\title{
G-Networks: Multiple Classes of Positive Customers, Signals, and Product Form Results
}

\author{
Erol Gelenbe \\ School of Electrical Engineering and Computer Science \\ University of Central Florida \\ Orlando, FL 32816 \\ erol@cs.ucf.edu
}

\begin{abstract}
The purpose of this tutorial presentation is to introduce GNetworks, or Gelenbe Networks, which are product form queueing networks which include normal or positive customers, as well as negative customers which destroy other customers, and triggers which displace other customers from one queue to another. We derive the balance equations for these models in the context of multiple customer classes, show the product form results, and exhibit the traffic equations which - in this case, contrary to BCMP and Jackson networks - are non-linear. This leads to interesting issues of existence and uniqueness of the steady-state solution. Gelenbe Network can be used to model large scale computer systems and networks in which signaling functions represented by negative customers and triggers are used to achieve flow and congestion control.
\end{abstract}

\section{Introduction}

In this survey and tutorial, we discuss a class of queueing networks, originally inspired by our work on neural networks, in which customers are either "signals" or positive customers.

Positive customers enter a queue and receive service as ordinary queueing network customers; they constitute queue length. A signal may be of a "negative customer", or it may be a "trigger". Signals do not receive service, and disappear after having visited a queue. If the signal is a trigger, then it actually transfers a customer from the queue it arrives to, to some other queue according to a probabilistic rule. On the other hand, a negative customer simply depletes the length of the queue to which it arrives if the queue is non-empty. One can also consider that a negative customer is a special kind of trigger which simply sends a customer to the "outside world" rather than transferring it to another queue. Positive customers which leave a queue to enter another queue can become signals or remain positive customers.

Additional primitive operations for these networks have also been introduced in [12]. The computation of numerical solutions to the non-linear traffic equations of some of these models have been discussed in [6]. Applications to networking problems are reported in 17. A model of doubly redundant systems using Gnetworks, where work is scheduled on two different processors and then cancelled 
at one of the processors if the work is successfully completed at the other, is presented in [7. The extension of the original model with positive and signals [4] to multiple classes was proposed and obtained in various papers 911/1519.

Some early neural network applications of G-networks are summarized in a survey article [18. From the neural network approach, the model in 9] was applied to texture generation in colour images in an early paper [10].

The present survey Includes the results presented in [20, where multiple classes of positive and signals are discussed, and we also include multiple classes of triggers. Thus in this paper we discuss G-Networks with multiple classes of positive customers and one or more classes of signals.

Three types of service centers with their corresponding service disciplines are examined:

- Type 1 : first-in-first-out (FIFO),

- Type 2 : processor sharing (PS),

- Type 4 : last-in-first-out with preemptive resume priority (LIFO/PR).

With reference to the usual terminology related to the BCMP theorem [2], we exclude from the present discussion the Type 3 service centers with an infinite number of servers since they will not be covered by our results. Furthermore, in this paper we deal only with exponentially distributed service times.

In Section 2 we will prove that these multiple class G-Networks, with Type 1,2 and 4 service centers, have product form.

\section{The Model}

We consider networks with an arbitrary number $n$ of queues, an arbitrary number of positive customer classes $K$, and an arbitrary number of signal classes $S$. External arrival streams to the network are independent Poisson processes for positive customers of some class $k$ and signals of some class $c$. We denote by $\Lambda_{i, k}$ the external arrival rate of positive customers of class $k$ to queue $i$ and by $\lambda_{i, m}$ be the external arrival rate of signals of class $m$ to queue $i$.

Only positive customers are served, and after service they may change class, service center and nature (positive signal), or depart from the system. The movement of customers between queues, classes and nature (positive to signal) is represented by a Markov chain.

At its arrival in a non-empty queue, a signal selects a positive customer as its "target" in the queue in accordance with the service discipline at this station. If the queue is empty, then the signalsimply disappears. Once the target is selected, the signaltries to trigger the movement of the selected customer. A negative customer, of some class $m$, succeeds in triggering the movement of the selected positive customer of some class $k$, at service center $i$ with probability $K_{i, m, k}$. With probability $\left(1-K_{i, m, k}\right)$ it does not succeed. A signal disappears as soon as it tries to trigger the movement of its targeted customer. Recall that 
signal is either exogenous, or is obtained by the transformation of a positive customer as it leaves a queue.

A positive customer of class $k$ which leaves queue $i$ (after finishing service) goes to queue $j$ as a positive customer of class $l$ with probability $P^{+}[i, j][k, l]$, or as a signal of class $m$ with probability $P^{-}[i, j][k, m]$. It may also depart from the network with probability $d[i, k]$. Obviously we have for all $i, k$ :

$$
\sum_{j=1}^{n} \sum_{l=1}^{R} P^{+}[i, j][k, l]+\sum_{j=1}^{n} \sum_{m=1}^{S} P^{-}[i, j][k, m]+d[i, k]=1
$$

We assume that all service centers have exponential service time distributions. In the three types of service centers, each class of positive customers may have a distinct service rate

rik. When the service center is of Type 1 (FIFO) we place the following constraint on the service rate and the movement triggering rate due to incoming signals:

$$
r i k+\sum_{m=1}^{S} K_{i, m, k} \lambda_{i, m}=c_{i}
$$

Note that this constraint, together with the constraint (3) given below, have the effect of producing a single positive customer class equivalent for service centers with FIFO discipline. The following constraints on the movement triggering probability are assumed to exist. Note that because services are exponentially distributed, positive customers of a given class are indistinguishable for movement triggering because of the Markovian property of service time.

- The following constraint must hold for all stations $i$ of Type 1 and classes of signals $m$ such that $\sum_{j=1}^{n} \sum_{l=1}^{R} P^{-}[j, i][l, m]>0$

$$
\text { for all classes of positive customers } a \text { and } b, K_{i, m, a}=K_{i, m, b}
$$

This constraint implies that a signal of some class $m$ arriving from the network does not "distinguish" between the positive customer classes it will try to trigger the movement, and that it will treat them all in the same manner.

- For a Type 2 server, the probability that any one positive customer of the queue is selected by the arriving signalis $1 / c$ if $c$ is the total number of customers in the queue.

For Type 1 service centers, one may consider the following conditions which are simpler than (2) and (3):

$$
\begin{gathered}
r_{i a}=r_{i b} \\
K_{i, m, a}=K_{i, m, b}
\end{gathered}
$$

for all classes of positive customers $a$ and $b$, and all classes of signals $m$. Note however that these new conditions are more restrictive, though they do imply that (2), (3) hold. 


\subsection{State Representation}

We denote the state at time $\mathrm{t}$ of the queueing network by a vector $x(t)=$ $\left(x_{1}(t), \ldots, x_{n}(t)\right)$. Here $x_{i}(t)$ represents the state of service center $\mathrm{i}$. The vector $x=\left(x_{1} \ldots, x_{n}\right)$ will denote a particular value of the state and $\left|x_{i}\right|$ will be the total number of customers in queue $i$ for state $x$.

For Type 1 and Type 4 servers, the instantaneous value of the state $x_{i}$ of queue $i$ is represented by the vector of elements whose length is the number of customers in the queue and whose $j t h$ element $x_{i, j}$ is the class index of the $j t h$ customer in the queue. Furthermore, the customers are ordered according to the service order (FIFO or LIFO); it is always the customer at the head of the list which is in service. We denote by $c_{i, 1}$ the class number of the customer in service and by $c_{i, \infty}$ the class number of the last customer in the queue.

For a PS (Type 2) service station, the instantaneous value of the state $x_{i}$ is represented by the vector $\left(x_{i, k}\right)$ which is the number of customers of class $k$ in queue $i$.

\section{Main Theorem}

Let $P(x)$ denote the stationary probability distribution of the state of the network. It is given by the following product form result.

Theorem 1 Consider a G-network with the restrictions and properties described in the previous sections. If the system of non-linear equations:

$$
\begin{aligned}
q_{i, k} & =\frac{\Lambda_{i, k}+\Lambda_{i, k}^{+}}{r_{i, k}+\sum_{m=1}^{S} K_{i, m, k}\left[\lambda_{i, m}+\lambda_{i, m}^{-}\right]} \\
\Lambda_{i, k}^{+} & =\sum_{j=1}^{n} \sum_{l=1}^{R} P^{+}[j, i][l, k] r_{j, l} q_{j, l} \\
& +\sum_{j=1}^{n} \sum_{l=1}^{R} \sum_{h=1}^{n} \sum_{m=1}^{S} \sum_{s=1}^{R} r_{j, l} q_{j, l} P^{-}[j, h][l, m] K_{h, m, s} q_{h, s} Q[h, i][s, k] \\
& +\sum_{j=1}^{n} \sum_{m=1}^{S} \sum_{s=1}^{R} \lambda_{j, m} K_{j, m, s} q_{j, s} Q[j, i][s, k] \\
\lambda_{i, m}^{-} & =\sum_{j=1}^{n} \sum_{l=1}^{R} P^{-}[j, i][l, m] r_{j, l} q_{j, l}
\end{aligned}
$$

has a solution such that for each pair $i, k: 0<q_{i, k}$ and for each station $i: \sum_{k=1}^{R} q_{i, k}<1$, then the stationary probability distribution of the network state is:

$$
P(x)=G \prod_{i=1}^{n} g_{i}\left(x_{i}\right)
$$


where each $g_{i}\left(x_{i}\right)$ depends on the type of service center $i$. The $g_{i}\left(x_{i}\right)$ in (5) have the following form :

FIFO. If the service center is of Type 1, then

$$
g_{i}\left(x_{i}\right)=\prod_{n=1}^{\left|x_{i}\right|} q_{i, v_{i, n}}
$$

PS. If the service center is of Type 2, then

$$
g_{i}\left(x_{i}\right)=\left|x_{i}\right| ! \prod_{k=1}^{R} \frac{\left(q_{i, k}\right)^{x_{i, k}}}{x_{i, k} !}
$$

LIFO/PR. If the service center is of Type 4 , then

$$
g_{i}\left(x_{i}\right)=\prod_{n=1}^{\left|x_{i}\right|} q_{i, v_{i, n}}
$$

and $G$ is the normalization constant.

Notice that $\Lambda_{i, k}^{+}$may be written as:

$$
\begin{aligned}
\Lambda_{i, k}^{+} & =\sum_{j=1}^{n} \sum_{l=1}^{R} r_{j, l} q_{j, l} P^{+}[j, i][l, k] \\
& +\sum_{j=1}^{n} \sum_{l=1}^{R} \sum_{m=1}^{S} q_{j, l} Q[j, i][l, k] K_{j, m, l}\left[\lambda_{j, m}+\lambda_{j, m}^{-}\right]
\end{aligned}
$$

The conditions requiring that $q_{i, k}>0$ and on that their sum over all classes at each center be less than 1 simply insure the existence of the normalizing constant $G$ in (8) and the stability of the network.

\section{Proof of the Main Result}

The proof follows the same lines as that for a similar result but more restrictive result in 20] which does not cover the case of triggers. The reader who is not interested in the technical details may prefer to skip this section. We begin with some technical Lemmas.

Lemma 1 The following flow equation is satisfied:

$$
\begin{aligned}
& \sum_{i=1}^{n} \sum_{k=1}^{R} \Lambda_{i, k}^{+}+\sum_{i=1}^{n} \sum_{m=1}^{S} \lambda_{i, m}^{-} \\
& =\sum_{i=1}^{n} \sum_{k=1}^{R} q_{i, k} r_{i, k}(1-d[i, k]) \\
& +\sum_{i=1}^{n} \sum_{k=1}^{R} \sum_{j=1}^{n} \sum_{l=1}^{R} \sum_{m=1}^{S} q_{j, l} Q[j, i][l, k] K_{j, m, l}\left[\lambda_{j, m}+\lambda_{j, m}^{-}\right]
\end{aligned}
$$


Proof : Consider (12), then sum it for all the stations and all the classes and exchange the order of summations in the right-hand side of the equation :

$$
\begin{aligned}
\sum_{i=1}^{n} \sum_{k=1}^{R} \Lambda_{i, k}^{+} & =\sum_{j=1}^{n} \sum_{l=1}^{R} r_{j, l} q_{j, l}\left(\sum_{i=1}^{n} \sum_{k=1}^{R} P^{+}[j, i][l, k]\right) \\
& +\sum_{i=1}^{n} \sum_{k=1}^{R} \sum_{j=1}^{n} \sum_{l=1}^{R} \sum_{m=1}^{S} q_{j, l} Q[j, i][l, k] K_{j, m, l}\left[\lambda_{j, m}+\lambda_{j, m}^{-}\right]
\end{aligned}
$$

Similarly, using equation (7)

$$
\sum_{i=1}^{n} \sum_{m=1}^{S} \lambda_{i, m}^{-}=\sum_{j=1}^{n} \sum_{l=1}^{R} r_{j, l} q_{j, l}\left(\sum_{i=1}^{n} \sum_{m=1}^{S} P^{-}[j, i][l, m]\right)
$$

Furthermore:

$$
\begin{aligned}
& \sum_{i=1}^{n} \sum_{k=1}^{R} \Lambda_{i, k}^{+}+\sum_{i=1}^{n} \sum_{m=1}^{S} \lambda_{i, m}^{-} \\
& =\sum_{j=1}^{n} \sum_{l=1}^{R} r_{j, l} q_{j, l}\left(\sum_{i=1}^{n} \sum_{k=1}^{R} P^{+}[j, i][l, k]+\sum_{i=1}^{n} \sum_{m=1}^{S} P^{-}[j, i][l, m]\right) \\
& +\sum_{i=1}^{n} \sum_{k=1}^{R} \sum_{j=1}^{n} \sum_{l=1}^{R} \sum_{m=1}^{S} q_{j, l} Q[j, i][l, k] K_{j, m, l}\left[\lambda_{j, m}+\lambda_{j, m}^{-}\right]
\end{aligned}
$$

According to the definition of the routing matrix $P$ (equation (1)), we have

$$
\begin{aligned}
& \sum_{i=1}^{n} \sum_{k=1}^{R} \Lambda_{i, k}^{+}+\sum_{i=1}^{n} \sum_{m=1}^{S} \lambda_{i, m}^{-} \\
& =\sum_{j=1}^{n} \sum_{l=1}^{R} r_{j, l} q_{j, l}(1-d[j, l]) \\
& \quad+\sum_{i=1}^{n} \sum_{k=1}^{R} \sum_{j=1}^{n} \sum_{l=1}^{R} \sum_{m=1}^{S} q_{j, l} Q[j, i][l, k] K_{j, m, l}\left[\lambda_{j, m}+\lambda_{j, m}^{-}\right]
\end{aligned}
$$

Thus the proof of the Lemma is complete.

In order to carry out the algebraic manipulations of the stationary ChapmanKolmogorov (global balance) equations, we introduce some notation and develop intermediate results:

- The state dependent service rates for customers at service center $j$ will be denoted by $M_{j, l}\left(x_{j}\right)$ where $x_{j}$ refers to the state of the service center and $l$ is the class of the customer concerned. From the definition of the service rate $r j l$, we obtain for the three types of stations : 
FIFO and LIFO/PR $M_{j, l}\left(x_{j}\right)=r_{j, l} 1_{\left\{c_{j, 1}=l\right\}}$,

PS $M_{j, l}\left(x_{j}\right)=r_{j, l} \frac{x_{j, l}}{\left|x_{j}\right|}$.

- $N_{j, l}\left(x_{j}\right)$ is the movement triggering rate of class $l$ positive customers due to external arrivals of all the classes of signals:

FIFO and LIFO/PR $N_{j, l}\left(x_{j}\right)=1_{\left\{c_{j, 1}=l\right\}} \sum_{m=1}^{S} K_{j, m, l} \lambda_{j, m}$ PS $N_{j, l}\left(x_{j}\right)=\frac{x_{j, l}}{\left|x_{j}\right|} \sum_{m=1}^{S} K_{j, m, l} \lambda_{j, m}$.

- $A_{j, l}\left(x_{j}\right)$ is the condition which establishes that it is possible to reach state $x_{j}$ by an arrival of a positive customer of class $l$

FIFO $A_{j, l}\left(x_{j}\right)=1_{\left\{c_{j, \infty}=l\right\}}$,

LIFO/PR $A_{j, l}\left(x_{j}\right) \stackrel{1}{=} 1_{\left\{c_{j, 1}=l\right\}}$,

PS $A_{j, l}\left(x_{j}\right)=1_{\left\{\left|x_{j, l}\right|>0\right\}}$.

- $Z_{j, l, m}\left(x_{j}\right)$ is the probability that a signal of class $m$, arriving from the network, will trigger the movement of a positive customer of class $l$.

FIFO and LIFO/PR $Z_{j, l, m}\left(x_{j}\right)=1_{\left\{c_{j, 1}=l\right\}} K_{j, m, l}$

PS $Z_{j, l, m}\left(x_{j}\right)=\frac{x_{j, l}}{\left|x_{j}\right|} K_{j, m, l}$.

- $Y_{j, m}\left(x_{j}\right)$ is the probability that a signal of class $m$ which enters a non empty queue, will not trigger the movement of a positive customer.

FIFO and LIFO/PR $Y_{j, m}\left(x_{j}\right)=\sum_{l=1}^{R} 1_{\left\{c_{j, 1}=l\right\}}\left(1-K_{j, m, l}\right)$

PS $Y_{j, m}\left(x_{j}\right)=\sum_{l=1}^{R}\left(1-K_{j, m, l}\right) \frac{x_{j, l}}{\left|x_{j}\right|}$.

Denote by $\left(x_{j}+e_{j, l}\right)$ the state of station $j$ obtained by adding to the $j-$ th queue a positive customer of class $l$. Let $\left(x_{i}-e_{i, k}\right)$ be the state obtained by removing from the end of the list of customers in queue, a class $k$ customer if it is there; otherwise $\left(x_{i}-e_{i, k}\right)$ is not defined.

Lemma 2 For any Type 1, 2, or 4 service center, the following relations hold:

$$
\begin{aligned}
M_{j, l}\left(x_{j}+e_{j, l}\right) \frac{g_{j}\left(x_{j}+e_{j, l}\right)}{g_{j}\left(x_{j}\right)} & =r_{j, l} q_{j, l} \\
N_{j, l}\left(x_{j}+e_{j, l}\right) \frac{g_{j}\left(x_{j}+e_{j, l}\right)}{g_{j}\left(x_{j}\right)} & =\sum_{m=1}^{S}\left(K_{j, m, l} \lambda_{j, m}\right) q_{j, l} \\
Z_{j, l, m}\left(x_{j}+e_{j, l}\right) \frac{g_{j}\left(x_{j}+e_{j, l}\right)}{g_{j}\left(x_{j}\right)} & =K_{j, m, l} q_{j, l}
\end{aligned}
$$

The proof is purely algebraic.

Remark : As a consequence, we have from equations (12), (17) and (13):

$$
\begin{aligned}
\Lambda_{i, k}^{+} & =\sum_{j=1}^{n} \sum_{l=1}^{R} M_{j, l}\left(x_{j}+e_{j, l}\right) \frac{g_{j}\left(x_{j}+e_{j, l}\right)}{g_{j}\left(x_{j}\right)} P^{+}[j, i][l, k] \\
& +\sum_{j=1}^{n} \sum_{l=1}^{R} \sum_{m=1}^{S} q_{j, l} Q[j, i][l, k] K_{j, m, l}\left[\lambda_{j, m}+\lambda_{j, m}^{-}\right]
\end{aligned}
$$

and 


$$
\lambda_{i, m}^{-}=\sum_{j=1}^{n} \sum_{l=1}^{R} M_{j, l}\left(x_{j}+e_{j, l}\right) \frac{g_{j}\left(x_{j}+e_{j, l}\right)}{g_{j}\left(x_{j}\right)} P^{-}[j, i][l, m]
$$

Lemma 3 Let $i$ be any Type 1, 2, or 4 station, and let $\Delta_{i}\left(x_{i}\right)$ be:

$$
\begin{aligned}
& \Delta_{i}\left(x_{i}\right)=\sum_{m=1}^{S} \lambda_{i, m}^{-} Y_{i, m}\left(x_{i}\right) \\
& -\sum_{k=1}^{R}\left(M_{i, k}\left(x_{i}\right)+N_{i, k}\left(x_{i}\right)\right) \\
& +\sum_{k=1}^{R} A_{i, k}\left(x_{i}\right)\left(\Lambda_{i, k}+\Lambda_{i, k}^{+}\right) \frac{g_{i}\left(x_{i}-e_{i, k}\right)}{g_{i}\left(x_{i}\right)}
\end{aligned}
$$

Then for the three types of service centers, $1_{\left\{\left|x_{i}\right|>0\right\}} \Delta_{i}\left(x_{i}\right)=\sum_{m=1}^{S} \lambda_{i, m}^{-}$ $1_{\left\{\left|x_{i}\right|>0\right\}}$.

The proof of Lemma 3 is in a separate subsection at the end of this paper in order to make the text somewhat easier to follow.

Let us now turn to the proof of Theorem 1. The global balance equation of the networks which are considered is:

$$
\begin{aligned}
& P(x)\left[\sum_{j=1}^{n} \sum_{l=1}^{R}\left(\Lambda_{j, l}+M_{j, l}\left(x_{j}\right) 1_{\left\{\left|x_{j}\right|>0\right\}}+N_{j, l}\left(x_{j}\right) 1_{\left\{\left|x_{j}\right|>0\right\}}\right)\right] \\
& =\sum_{j=1}^{n} \sum_{l=1}^{R} P\left(x-e_{j, l}\right) \Lambda_{j, l} A_{j, l}\left(x_{j}\right) 1_{\left\{\left|x_{j}\right|>0\right\}} \\
& +\sum_{j=1}^{n} \sum_{l=1}^{R} P\left(x+e_{j, l}\right) N_{j, l}\left(x_{j}+e_{j, l}\right) D[j, l] \\
& +\sum_{j=1}^{n} \sum_{l=1}^{R} P\left(x+e_{j, l}\right) M_{j, l}\left(x_{j}+e_{j, l}\right) d[j, l] \\
& +\sum_{i=1}^{n} \sum_{j=1}^{n} \sum_{l=1}^{R} \sum_{m=1}^{S} M_{j, l}\left(x_{j}+e_{j, l}\right) P\left(x+e_{j, l}\right) P^{-}[j, i][l, m] Y_{i, m}\left(x_{i}\right) 1_{\left\{\left|x_{i}\right|>0\right\}} \\
& +\sum_{i=1}^{n} \sum_{j=1}^{n} \sum_{l=1}^{R} \sum_{m=1}^{S} M_{j, l}\left(x_{j}+e_{j, l}\right) P\left(x+e_{j, l}\right) P^{-}[j, i][l, m] 1_{\left\{\left|x_{i}\right|=0\right\}} \\
& +\sum_{i=1}^{n} \sum_{j=1}^{n} \sum_{k=1}^{R} \sum_{l=1}^{R} M_{j, l}\left(x_{j}+e_{j, l}\right) P\left(x-e_{i, k}+e_{j, l}\right) P^{+}[j, i][l, k] A_{i, k}\left(x_{i}\right) 1_{\left\{\left|x_{i}\right|>0\right\}}
\end{aligned}
$$




$$
\begin{aligned}
& +\sum_{i=1}^{n} \sum_{j=1}^{n} \sum_{k=1}^{R} \sum_{l=1}^{R} N_{j, l}\left(x_{j}+e_{j, l}\right) P\left(x-e_{i, k}+e_{j, l}\right) Q[j, i][l, k] A_{i, k}\left(x_{i}\right) 1_{\left\{\left|x_{i}\right|>0\right\}} \\
& +\sum_{i=1}^{n} \sum_{j=1}^{n} \sum_{k=1}^{R} \sum_{l=1}^{R} \sum_{m=1}^{S} M_{j, l}\left(x_{j}+e_{j, l}\right) P\left(x+e_{i, k}+e_{j, l}\right) P^{-}[j, i][l, m] Z_{i, k, m} \\
& +\sum_{i=1}^{n} \sum_{j=1}^{n} \sum_{k=1}^{R} \sum_{l=1}^{R} \sum_{m=1}^{S} \sum_{h=1}^{n} \sum_{s=1}^{R}\left(x_{i}+e_{i, k}\right) D[i, k] \\
& \left.\quad Z_{i, k, m}\left(x_{i}+e_{i, k}\right) Q[i, h][k, s] A_{h, s}\left(x_{h}\right) 1_{\left\{\left|x_{h}\right|>0\right\}}\right)
\end{aligned}
$$

We divide both sides by $P(x)$, assume that there is a product form solution, and apply Lemma 2 .

$$
\begin{aligned}
& \sum_{j=1}^{n} \sum_{l=1}^{R}\left(\Lambda_{j, l}+M_{j, l}\left(x_{j}\right) 1_{\left\{\left|x_{j}\right|>0\right\}}+N_{j, l}\left(x_{j}\right) 1_{\left\{\left|x_{j}\right|>0\right\}}\right) \\
& =\sum_{j=1}^{n} \sum_{l=1}^{R} \frac{g_{j}\left(x_{j}-e_{j, l}\right)}{g_{j}\left(x_{j}\right)} \Lambda_{j, l} A_{j, l}\left(x_{j}\right) 1_{\left\{\left|x_{j}\right|>0\right\}} \\
& +\sum_{j=1}^{n} \sum_{l=1}^{R} \sum_{m=1}^{S} \lambda_{j, m} K_{j, m, l} q_{j, l} D[j, l]+\sum_{j=1}^{n} \sum_{l=1}^{R} r_{j, l} q_{j, l} d[j, l] \\
& +\sum_{i=1}^{n} \sum_{j=1}^{n} \sum_{l=1}^{R} \sum_{m=1}^{S} r_{j, l} q_{j, l} P^{-}[j, i][l, m] Y_{i, m}\left(x_{i}\right) 1_{\left\{\left|x_{i}\right|>0\right\}} \\
& +\sum_{i=1}^{n} \sum_{j=1}^{n} \sum_{l=1}^{R} \sum_{m=1}^{S} r_{j, l} q_{j, l} P^{-}[j, i][l, m] 1_{\left\{\left|x_{i}\right|=0\right\}} \\
& +\sum_{i=1}^{n} \sum_{j=1}^{n} \sum_{k=1}^{R} \sum_{l=1}^{R} r_{j, l} q_{j, l} P^{+}[j, i][l, k] A_{i, k}\left(x_{i}\right) \frac{g_{i}\left(x_{i}-e_{i, k}\right)}{g_{i}\left(x_{i}\right)} 1_{\left\{\left|x_{i}\right|>0\right\}} \\
& +\sum_{i=1}^{n} \sum_{j=1}^{n} \sum_{k=1}^{R} \sum_{l=1}^{R} \sum_{m=1}^{S} \lambda_{j, m} K_{j, m, l} q_{j, l} Q[j, i][l, k] A_{i, k}\left(x_{i}\right) \frac{g_{i}\left(x_{i}-e_{i, k}\right)}{g_{i}\left(x_{i}\right)} 1_{\left\{\left|x_{i}\right|>0\right\}} \\
& +\sum_{i=1}^{n} \sum_{j=1}^{n} \sum_{k=1}^{R} \sum_{l=1}^{R} \sum_{m=1}^{S} r_{j, l} q_{j, l} P^{-}[j, i][l, m] K_{i, m, k} q_{i, k} D[i, k] \\
& +
\end{aligned}
$$


We now apply (7) to the fourth, fifth, eigth and ninth terms of the second member of the equation:

$$
\begin{aligned}
\sum_{j=1}^{n} & \sum_{l=1}^{R}\left(\Lambda_{j, l}+M_{j, l}\left(x_{j}\right) 1_{\left\{\left|x_{j}\right|>0\right\}}+N_{j, l}\left(x_{j}\right) 1_{\left\{\left|x_{j}\right|>0\right\}}\right) \\
= & \sum_{j=1}^{n} \sum_{l=1}^{R} \frac{g_{j}\left(x_{j}-e_{j, l}\right)}{g_{j}\left(x_{j}\right)} \Lambda_{j, l} A_{j, l}\left(x_{j}\right) 1_{\left\{\left|x_{j}\right|>0\right\}} \\
& +\sum_{j=1}^{n} \sum_{l=1}^{R} \sum_{m=1}^{S} \lambda_{j, m} K_{j, m, l} q_{j, l} D[j, l]+\sum_{j=1}^{n} \sum_{l=1}^{R} r_{j, l} q_{j, l} d[j, l] \\
& +\sum_{i=1}^{n} \sum_{m=1}^{S} \lambda_{i, m}^{-} Y_{i, m}\left(x_{i}\right) 1_{\left\{\left|x_{i}\right|>0\right\}} \\
& +\sum_{i=1}^{n} \sum_{m=1}^{S} \lambda_{i, m}^{-} 1_{\left\{\left|x_{i}\right|=0\right\}} \\
& +\sum_{i=1}^{n} \sum_{j=1}^{n} \sum_{k=1}^{R} \sum_{l=1}^{R} r_{j, l} q_{j, l} P^{+}[j, i][l, k] A_{i, k}\left(x_{i}\right) \frac{g_{i}\left(x_{i}-e_{i, k}\right)}{g_{i}\left(x_{i}\right)} 1_{\left\{\left|x_{i}\right|>0\right\}} \\
& +\sum_{i=1}^{n} \sum_{j=1}^{n} \sum_{k=1}^{R} \sum_{l=1}^{R} \sum_{m=1}^{S} \lambda_{j, m} K_{j, m, l} q_{j, l} Q[j, i][l, k] A_{i, k}\left(x_{i}\right) \frac{g_{i}\left(x_{i}-e_{i, k}\right)}{g_{i}\left(x_{i}\right)} 1_{\left\{\left|x_{i}\right|>0\right\}} \\
& +\sum_{i=1}^{n} \sum_{k=1}^{R} \sum_{m=1}^{S} \lambda_{i, m}^{-} K_{i, m, k} q_{i, k} D[i, k] \\
& +\sum_{i=1}^{n} \sum_{j=1}^{n} \sum_{l=1}^{R} \sum_{k=1}^{R} \sum_{m=1}^{S} \lambda_{j, m}^{-} K_{j, m, l} q_{j, l} Q[j, i][l, k] \frac{g_{i}\left(x_{i}-e_{i, k}\right)}{g_{i}\left(x_{i}\right)} A_{i, k}\left(x_{i}\right) 1_{\left\{\left|x_{i}\right|>0\right\}}
\end{aligned}
$$

We group the first, sixth, seventh and ninth terms of the right side of the equation, and pass the two last terms of the first member to the second:

$$
\begin{aligned}
& \sum_{j=1}^{n} \sum_{l=1}^{R}\left(\Lambda_{j, l}\right) \\
& =-\sum_{i=1}^{n} \sum_{k=1}^{R}\left(M_{i, k}\left(x_{i}\right)+N_{i, k}\left(x_{i}\right)\right) 1_{\left\{\left|x_{i}\right|>0\right\}} \\
& +\sum_{i=1}^{n} \sum_{k=1}^{R} \frac{g_{i}\left(x_{i}-e_{i, k}\right)}{g_{i}\left(x_{i}\right)} A_{i, k}\left(x_{i}\right) 1_{\left\{\left|x_{i}\right|>0\right\}}\left(\Lambda_{i, k}+\Lambda_{i, k}^{+}\right) \\
& +\sum_{i=1}^{n} \sum_{m=1}^{S} \lambda_{i, m}^{-} Y_{i, m}\left(x_{i}\right) 1_{\left\{\left|x_{i}\right|>0\right\}}
\end{aligned}
$$




$$
\begin{aligned}
& +\sum_{j=1}^{n} \sum_{l=1}^{R} \sum_{m=1}^{S} \lambda_{j, m} K_{j, m, l} q_{j, l} D[j, l]+\sum_{j=1}^{n} \sum_{l=1}^{R} r_{j, l} q_{j, l} d[j, l] \\
& +\sum_{i=1}^{n} \sum_{m=1}^{S} \lambda_{i, m}^{-} 1_{\left\{\left|x_{i}\right|=0\right\}} \\
& +\sum_{i=1}^{n} \sum_{k=1}^{R} \sum_{m=1}^{S} \lambda_{i, m}^{-} K_{i, m, k} q_{i, k} D[i, k]
\end{aligned}
$$

We now apply Lemma 3 to the sum of the three first terms of the second equation:

$$
\begin{aligned}
& \sum_{j=1}^{n} \sum_{l=1}^{R} \Lambda_{j, l} \\
& =\sum_{i=1}^{n} \sum_{m=1}^{S} \lambda_{i, m}^{-} 1_{\left\{\left|x_{i}\right|>0\right\}} \\
& +\sum_{j=1}^{n} \sum_{l=1}^{R} \sum_{m=1}^{S} \lambda_{j, m} K_{j, m, l} q_{j, l} D[j, l]+\sum_{j=1}^{n} \sum_{l=1}^{R} r_{j, l} q_{j, l} d[j, l] \\
& +\sum_{i=1}^{n} \sum_{m=1}^{S} \lambda_{i, m}^{-} 1_{\left\{\left|x_{i}\right|=0\right\}} \\
& +\sum_{j=1}^{n} \sum_{k=1}^{R} \sum_{m=1}^{S} \lambda_{j, m}^{-} K_{j, m, k} q_{j, k} D[j, k]
\end{aligned}
$$

Now we group the first and fourth terms, and the second and fifth terms of the right side of the equation.

$$
\begin{aligned}
& \sum_{j=1}^{n} \sum_{l=1}^{R} \Lambda_{j, l} \\
& =\sum_{i=1}^{n} \sum_{m=1}^{S} \lambda_{i, m}^{-} \\
& +\sum_{j=1}^{n} \sum_{l=1}^{R} \sum_{m=1}^{S} q_{j, l} K_{j, m, l}\left(\lambda_{j, m}+\lambda_{j, m}^{-}\right) D[j, l] \\
& +\sum_{j=1}^{n} \sum_{l=1}^{R} r_{j, l} q_{j, l} d[j, l]
\end{aligned}
$$

Substituting the value of $D[j, l]$ and the value of $d[j, l]$,

$$
\sum_{j=1}^{n} \sum_{l=1}^{R} \Lambda_{j, l}
$$




$$
\begin{aligned}
& =\sum_{i=1}^{n} \sum_{m=1}^{S} \lambda_{i, m}^{-}+\sum_{j=1}^{n} \sum_{l=1}^{R} \sum_{m=1}^{S} q_{j, l} K_{j, m, l}\left(\lambda_{j, m}+\lambda_{j, m}^{-}\right)+\sum_{j=1}^{n} \sum_{l=1}^{R} q_{j, l} r_{j, l} \\
& -\left(\sum_{i=1}^{n} \sum_{j=1}^{n} \sum_{l=1}^{R} \sum_{k=1}^{R} \sum_{m=1}^{S} q_{j, l} K_{j, m, l} Q[j, i][l, k]\left(\lambda_{j, m}+\lambda_{j, m}^{-}\right)\right. \\
& \left.+\sum_{i=1}^{n} \sum_{j=1}^{n} \sum_{l=1}^{R} \sum_{k=1}^{R} r_{j, l} q_{j, l} P^{+}[j, i][l, k]\right) \\
& -\sum_{i=1}^{n} \sum_{j=1}^{n} \sum_{l=1}^{R} \sum_{m=1}^{S} q_{j, l} r_{j, l} P^{-}[j, i][l, m]
\end{aligned}
$$
have:

and substituting for $q_{j l}$ in the second and third terms and grouping them we

$$
\begin{aligned}
& \sum_{j=1}^{n} \sum_{l=1}^{R} \Lambda_{j, l} \\
& =\sum_{i=1}^{n} \sum_{m=1}^{S} \lambda_{i, m}^{-} \\
& +\sum_{j=1}^{n} \sum_{l=1}^{R} \Lambda_{j, l}+\sum_{j=1}^{n} \sum_{l=1}^{R} \Lambda_{j, l}^{+} \\
& -\sum_{j=1}^{n} \sum_{l=1}^{R} \Lambda_{j, l}^{+}-\sum_{i=1}^{n} \sum_{m=1}^{S} \lambda_{i, m}^{-}
\end{aligned}
$$

which yields thefollowing equality which is obviously satisfied,

$$
\sum_{j=1}^{n} \sum_{l=1}^{R} \Lambda_{j, l}=\sum_{j=1}^{n} \sum_{l=1}^{R} \Lambda_{j, l}
$$

concluding the proof.

As in the BCMP [2] theorem, we can also compute the steady state distribution of the number of customers of each class in each queue. Let $y_{i}$ be the vector whose elements are $\left(y_{i, k}\right)$ the number of customers of class $k$ in station $i$. Let $y$ be the vector of vectors $\left(y_{i}\right)$. We omit the proof of the following result.

Theorem 2 If the system of equations (5), (6) and (7) has a solution then, the steady state distribution $\pi(y)$ is given by

$$
\pi(y)=\prod_{i=1}^{n} h_{i}\left(y_{i}\right)
$$

where the marginal probabilities $h_{i}\left(y_{i}\right)$ have the following form :

$$
h_{i}\left(y_{i}\right)=\left(1-\sum_{k=1}^{R} q_{i, k}\right)\left|y_{i}\right| ! \prod_{k=1}^{R}\left[\left(q_{i, k}\right)^{y_{i, k}} / y_{i, k} !\right]
$$




\subsection{Proof of Lemma 3}

The proof of Lemma 3 consists in algebraic manipulations of the terms in the balance equations related to each og the the three types of stations.

$L I F O / P R$. First consider an arbitrary LIFO station and recall the definition of $\Delta_{i}$ :

$$
\begin{aligned}
1_{\left\{\left|x_{i}\right|>0\right\}} \Delta_{i}\left(x_{i}\right) & =1_{\left\{\left|x_{i}\right|>0\right\}} \sum_{k=1}^{R} A_{i, k}\left(x_{i}\right)\left(\Lambda_{i, k}+\Lambda_{i, k}^{+}\right) \frac{g_{i}\left(x_{i}-e_{i, k}\right)}{g_{i}\left(x_{i}\right)} \\
& -1_{\left\{\left|x_{i}\right|>0\right\}} \sum_{k=1}^{R} M_{i, k}\left(x_{i}\right)-1_{\left\{\left|x_{i}\right|>0\right\}} \sum_{k=1}^{R} N_{i, k}\left(x_{i}\right) \\
& +1_{\left\{\left|x_{i}\right|>0\right\}} \sum_{m=1}^{S} \lambda_{i, m}^{-} Y_{i, m}\left(x_{i}\right)
\end{aligned}
$$

We substitute the values of $Y_{i, m}, M_{i, k}, N_{i, k}$ and $A_{i, k}$ for a LIFO station:

$$
\begin{aligned}
1_{\left\{\left|x_{i}\right|>0\right\}} \Delta_{i}\left(x_{i}\right) & =1_{\left\{\left|x_{i}\right|>0\right\}} \sum_{k=1}^{R} 1_{\left\{c_{i, 1}=k\right\}}\left(\Lambda_{i, k}+\Lambda_{i, k}^{+}\right) / q_{i, k} \\
& -1_{\left\{\left|x_{i}\right|>0\right\}} \sum_{k=1}^{R} 1_{\left\{c_{i, 1}=k\right\}} r_{i, k} \\
& -1_{\left\{\left|x_{i}\right|>0\right\}} \sum_{k=1}^{R} 1_{\left\{c_{i, 1}=k\right\}} \sum_{m=1}^{S} K_{i, m, k} \lambda_{i, m} \\
& +1_{\left\{\left|x_{i}\right|>0\right\}} \sum_{m=1}^{S} \lambda_{i, m}^{-} \sum_{k=1}^{R} 1_{\left\{c_{i, 1}=k\right\}}\left(1-K_{i, m, k}\right)
\end{aligned}
$$
obtain

$$
\begin{aligned}
1_{\left\{\left|x_{i}\right|>0\right\}} \Delta_{i}\left(x_{i}\right) & =1_{\left\{\left|x_{i}\right|>0\right\}} \sum_{k=1}^{R} 1_{\left\{c_{i, 1}=k\right\}}\left(\sum_{m=1}^{S} K_{i, m, k} \lambda_{i, m}^{-}+\sum_{m=1}^{S} \lambda_{i, m}^{-}\left(1-K_{i, m, k}\right)\right. \\
& =1_{\left\{\left|x_{i}\right|>0\right\}} \sum_{m=1}^{S} \lambda_{i, m}^{-} \sum_{k=1}^{R} 1_{\left\{c_{i, 1}=k\right\}}
\end{aligned}
$$

and as $1_{\left\{\left|x_{i}\right|>0\right\}} \sum_{k=1}^{R} 1_{\left\{c_{i, 1}=k\right\}}=1_{\left\{\left|x_{i}\right|>0\right\}}$, we finally get the result :

$$
1_{\left\{\left|x_{i}\right|>0\right\}} \Delta_{i}\left(x_{i}\right)=1_{\left\{\left|x_{i}\right|>0\right\}} \sum_{m=1}^{S} \lambda_{i, m}^{-}
$$


FIFO. Consider now an arbitrary FIFO station :

$$
\begin{aligned}
1_{\left\{\left|x_{i}\right|>0\right\}} \Delta_{i}\left(x_{i}\right) & =1_{\left\{\left|x_{i}\right|>0\right\}} \sum_{k=1}^{R} A_{i, k}\left(x_{i}\right)\left(\Lambda_{i, k}+\Lambda_{i, k}^{+}\right) \frac{g_{i}\left(x_{i}-e_{i, k}\right)}{g_{i}\left(x_{i}\right)} \\
& -1_{\left\{\left|x_{i}\right|>0\right\}} \sum_{k=1}^{R} M_{i, k}\left(x_{i}\right)-\sum_{k=1}^{R} 1_{\left\{\left|x_{i}\right|>0\right\}} N_{i, k}\left(x_{i}\right) \\
& +1_{\left\{\left|x_{i}\right|>0\right\}} \sum_{m=1}^{S} \lambda_{i, m}^{-} Y_{i, m}\left(x_{i}\right)
\end{aligned}
$$

Similarly, we substitute the values of $Y_{i, m}, M_{i, k}, N_{i, k}, A_{i, k}$ and $q_{i, k}$ :

$$
\begin{aligned}
1_{\left\{\left|x_{i}\right|>0\right\}} \Delta_{i}\left(x_{i}\right) & =1_{\left\{\left|x_{i}\right|>0\right\}} \sum_{k=1}^{R} 1_{\left\{c_{i, \infty}=k\right\}}\left(r_{i, k}+\sum_{m=1}^{S} K_{i, m, k} \lambda_{i, m}+\sum_{m=1}^{S} K_{i, m, k} \lambda_{i, m}^{-}\right) \\
& -1_{\left\{\left|x_{i}\right|>0\right\}} \sum_{k=1}^{R} 1_{\left\{c_{i, 1}=k\right\}} r_{i, k}-1_{\left\{\left|x_{i}\right|>0\right\}} \sum_{k=1}^{R} 1_{\left\{c_{i, 1}=k\right\}} \sum_{m=1}^{S} K_{i, m, k} \lambda_{i, m} \\
& +1_{\left\{\left|x_{i}\right|>0\right\}} \sum_{m=1}^{S} \lambda_{i, m}^{-} \sum_{k=1}^{R} 1_{\left\{c_{i, 1}=k\right\}}\left(1-K_{i, m, k}\right)
\end{aligned}
$$

We separate the last term into two parts, and regroup terms:

$$
\begin{aligned}
1_{\left\{\left|x_{i}\right|>0\right\}} \Delta_{i}\left(x_{i}\right) & =1_{\left\{\left|x_{i}\right|>0\right\}} \sum_{k=1}^{R} 1_{\left\{c_{i, \infty}=k\right\}}\left(r_{i, k}+\sum_{m=1}^{S} K_{i, m, k} \lambda_{i, m}+\sum_{m=1}^{S} K_{i, m, k} \lambda_{i, m}^{-}\right) \\
& -1_{\left\{\left|x_{i}\right|>0\right\}} \sum_{k=1}^{R} 1_{\left\{c_{i, 1}=k\right\}}\left(r_{i, k}+\sum_{m=1}^{S} K_{i, m, k} \lambda_{i, m}+\sum_{m=1}^{S} K_{i, m, k} \lambda_{i, m}^{-}\right) \\
& +1_{\left\{\left|x_{i}\right|>0\right\}} \sum_{m=1}^{S} \lambda_{i, m}^{-} \sum_{k=1}^{R} 1_{\left\{c_{i, 1}=k\right\}}
\end{aligned}
$$

Conditions (2) and (3) imply that the following relation must hold:

$$
\begin{gathered}
\sum_{k=1}^{R} 1_{\left\{c_{i, \infty}=k\right\}}\left(r_{i, k}+\sum_{m=1}^{S} K_{i, m, k} \lambda_{i, m}+\sum_{m=1}^{S} K_{i, m, k} \lambda_{i, m}^{-}\right)= \\
\sum_{k=1}^{R} 1_{\left\{c_{i, 1}=k\right\}}\left(r_{i, k}+\sum_{m=1}^{S} K_{i, m, k} \lambda_{i, m}+\sum_{m=1}^{S} K_{i, m, k} \lambda_{i, m}^{-}\right)
\end{gathered}
$$
result :

Thus, as $1_{\left\{\left|x_{i}\right|>0\right\}} \sum_{k=1}^{R} 1_{\left\{c_{i, 1}=k\right\}}=1_{\left\{\left|x_{i}\right|>0\right\}}$, we finally get the expected

$$
1_{\left\{\left|x_{i}\right|>0\right\}} \Delta_{i}\left(x_{i}\right)=1_{\left\{\left|x_{i}\right|>0\right\}} \sum_{m=1}^{S} \lambda_{i, m}^{-}
$$


$P S$. Consider now an arbitrary PS station :

$$
\begin{aligned}
1_{\left\{\left|x_{i}\right|>0\right\}} \Delta_{i}\left(x_{i}\right) & =1_{\left\{\left|x_{i}\right|>0\right\}} \sum_{k=1}^{R} A_{i, k}\left(x_{i}\right)\left(\Lambda_{i, k}+\Lambda_{i, k}^{+}\right) \frac{g_{i}\left(x_{i}-e_{i, k}\right)}{g_{i}\left(x_{i}\right)} \\
& -1_{\left\{\left|x_{i}\right|>0\right\}} \sum_{k=1}^{R} M_{i, k}\left(x_{i}\right)-\sum_{k=1}^{R} 1_{\left\{\left|x_{i}\right|>0\right\}} N_{i, k}\left(x_{i}\right) \\
& +1_{\left\{\left|x_{i}\right|>0\right\}} \sum_{m=1}^{S} \lambda_{i, m}^{-} Y_{i, m}\left(x_{i}\right)
\end{aligned}
$$

As usual, we substitute the values of $Y_{i, m}, M_{i, k}, N_{i, k}, A_{i, k}$ :

$$
\begin{aligned}
1_{\left\{\left|x_{i}\right|>0\right\}} \Delta_{i}\left(x_{i}\right) & =1_{\left\{\left|x_{i}\right|>0\right\}} \sum_{k=1}^{R} 1_{\left\{\left|x_{i, k}\right|>0\right\}} \frac{\left(\Lambda_{i, k}+\Lambda_{i, k}^{+}\right)}{q_{i, k}} \frac{x_{i, k}}{\left|x_{i}\right|} \\
& -1_{\left\{\left|x_{i}\right|>0\right\}} \sum_{k=1}^{R} r_{i, k} \frac{x_{i, k}}{\left|x_{i}\right|} \\
& -1_{\left\{\left|x_{i}\right|>0\right\}} \sum_{k=1}^{R} \frac{x_{i, k}}{\left|x_{i}\right|} \sum_{m=1}^{S} K_{i, m, k} \lambda_{i, m} \\
& +1_{\left\{\left|x_{i}\right|>0\right\}} \sum_{m=1}^{S} \sum_{k=1}^{R} \lambda_{i, m}^{-} \frac{x_{i, k}}{\left|x_{i}\right|}\left(1-K_{i, m, k}\right)
\end{aligned}
$$

Then, we apply equation (5) to substitute $q_{i, k}$. After some cancelations of terms we obtain :

$$
\begin{aligned}
1_{\left\{\left|x_{i}\right|>0\right\}} \Delta_{i}\left(x_{i}\right) & =1_{\left\{\left|x_{i}\right|>0\right\}} \sum_{k=1}^{R} \frac{x_{i, k}}{\left|x_{i}\right|} \sum_{m=1}^{S} K_{i, m, k} \lambda_{i, m}^{-} \\
& +1_{\left\{\left|x_{i}\right|>0\right\}} \sum_{m=1}^{S} \sum_{k=1}^{R} \lambda_{i, m}^{-} \frac{x_{i, k}}{\left|x_{i}\right|}\left(1-K_{i, m, k}\right)
\end{aligned}
$$

Finally we have:

$$
1_{\left\{\left|x_{i}\right|>0\right\}} \Delta_{i}\left(x_{i}\right)=1_{\left\{\left|x_{i}\right|>0\right\}} \sum_{k=1}^{R} \frac{x_{i, k}}{\left|x_{i}\right|} \sum_{m=1}^{S} \lambda_{i, m}^{-}
$$

Since $1_{\left\{\left|x_{i}\right|>0\right\}} \sum_{k=1}^{R} \frac{x_{i, k}}{\left|x_{i}\right|}=1_{\left\{\left|x_{i}\right|>0\right\}}$, once again, we establish the relation we need. This concludes the proof of Lemma 3

\section{References}

1. Kemmeny, J.G., Snell, J.L. "Finite Markov Chains", Von Nostrand, Princeton, 1965. 
2. Baskett F., Chandy K., Muntz R.R., Palacios F.G. "Open, closed and mixed networks of queues with different classes of customers", Journal ACM, Vol. 22, No 2, pp 248-260, April 1975.

3. Gelenbe E. "Random neural networks with negative and positive signals and product form solution", Neural Computation, Vol. 1, No. 4, pp 502-510, 1989.

4. Gelenbe E. "Product form queueing networks with negative and positive customers", Journal of Applied Probability, Vol. 28, pp 656-663, 1991.

5. Gelenbe E., Glynn P., Sigmann K. "Queues with negative customers", Journal of Applied Probability, Vol. 28, pp 245-250, 1991.

6. Fourneau J.M. "Computing the steady-state distribution of networks with positive and negative customers", Proc. 13-th IMACS World Congress on Computation and Applied Mathematics, Dublin, 1991.

7. E. Gelenbe, S. Tucci "Performances d'un système informatique dupliqué", Comptes-Rendus Acad. Sci., t 312, Série II, pp. 27-30, 1991.

8. Gelenbe E., Schassberger R. "Stability of G-Networks", Probability in the Engineering and Informational Sciences, Vol. 6, pp 271-276, 1992.

9. Fourneau, J.M., Gelenbe, E. "Multiple class G-networks," Conference of the ORSA Technical Committee on Computer Science, Williamsburg, VA, Balci, O. (Ed.), Pergamon, 1992.

10. Atalay, V., Gelenbe, E. "Parallel algorithm for colour texture generation using the random neural network model", International Journal of Pattern Recognition and Artificial Intelligence, Vol. 6, No. 2 \& 3, pp 437-446, 1992.

11. Miyazawa, M. "Insensitivity and product form decomposability of reallocatable GSMP", Advances in Applied Probability, Vol. 25, No. 2, pp 415-437, 1993.

12. Henderson, W. "Queueing networks with negative customers and negative queue lengths", Journal of Applied Probability, Vol. 30, No. 3, 1993.

13. Gelenbe E. "G-Networks with triggered customer movement", Journal of Applied Probability, Vol. 30, No. 3, pp 742-748, 1993.

14. Gelenbe E., "G-Networks with signals and batch removal", Probability in the Engineering and Informational Sciences, Vol. 7, pp 335-342, 1993.

15. Chao, X., Pinedo, M. "On generalized networks of queues with positive and negative arrivals", Probability in the Engineering and Informational Sciences, Vol. 7, pp 301-334, 1993.

16. Henderson, W., Northcote, B.S., Taylor, P.G. "Geometric equilibrium distributions for queues with interactive batch departures," Annals of Operations Research, Vol. 48, No. 1-4, 1994

17. Henderson, W., Northcote, B.S., Taylor, P.G. "Networks of customer queues and resource queues", Proc. International Teletraffic Congress 14, Labetoulle, J. and Roberts, J. (Eds.), pp 853-864, Elsevier, 1994.

18. Gelenbe, E. "G-networks: an unifying model for neural and queueing networks", Annals of Operations Research, Vol. 48, No. 1-4, pp 433-461, 1994.

19. Chao, X., Pinedo, M. "Product form queueing networks with batch services, signals, and product form solutions", Operations Research Letters, Vol. 17, pp 237242, 1995.

20. J.M. Fourneau, E. Gelenbe, R. Suros "G-networks with multiple classes of positive and negative customers," Theoretical Computer Science, Vol. 155, pp. 141-156, 1996.

21. Gelenbe, E., Labed A. "G-networks with multiple classes of customers and triggers", to appear. 